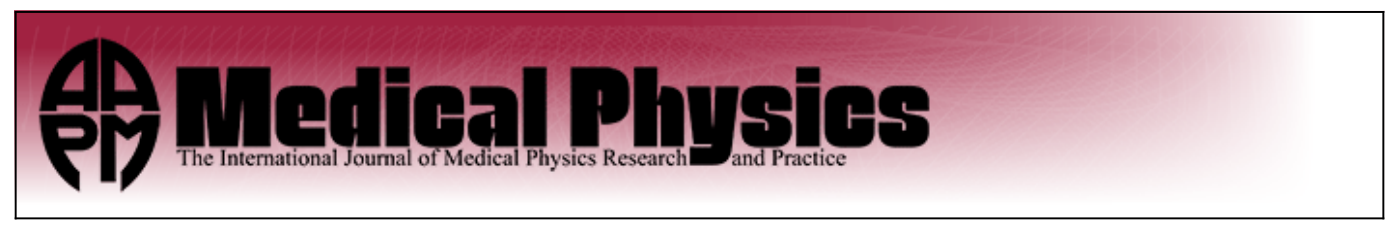

\title{
lon beam tracking using ultrasound motion detection
}

M. Prall, R. Kaderka, N. Saito, C. Graeff, C. Bert, M. Durante, K. Parodi, J. Schwaab, C. Sarti, and J. Jenne

Citation: Medical Physics 41, 041708 (2014); doi: 10.1118/1.4868459

View online: http://dx.doi.org/10.1118/1.4868459

View Table of Contents: http://scitation.aip.org/content/aapm/journal/medphys/41/4?ver=pdfcov

Published by the American Association of Physicists in Medicine

\section{Articles you may be interested in}

Combined magnetic resonance imaging and ultrasound echography guidance for motion compensated HIFU interventions

AIP Conf. Proc. 1503, 202 (2012); 10.1063/1.4769944

Comparison of a multileaf collimator tracking system and a robotic treatment couch tracking system for organ motion compensation during radiotherapy

Med. Phys. 39, 7032 (2012); 10.1118/1.4761868

Tumor tracking and motion compensation with an adaptive tumor tracking system (ATTS): System description and prototype testing

Med. Phys. 35, 3911 (2008); 10.1118/1.2964090

Dosimetric investigation of lung tumor motion compensation with a robotic respiratory tracking system: An experimental study

Med. Phys. 35, 1232 (2008); 10.1118/1.2842074

Accuracy of tumor motion compensation algorithm from a robotic respiratory tracking system: A simulation study Med. Phys. 34, 2774 (2007); 10.1118/1.2739811

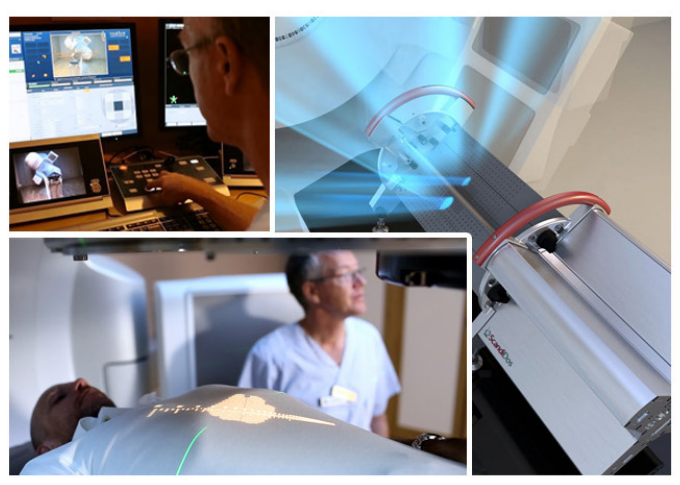

ScandiDos Delta 4 family offers precise and easy

QA from plan to the last fraction

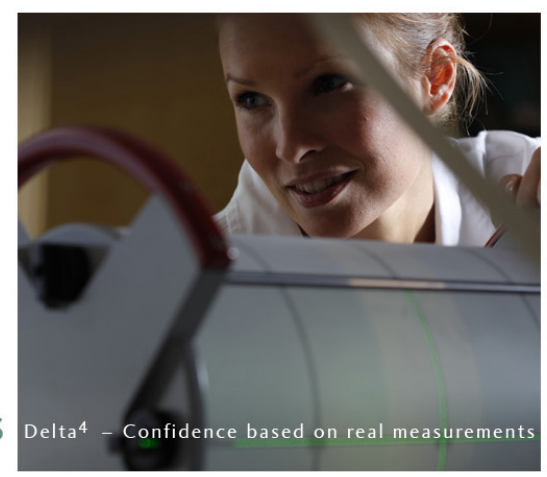




\title{
Ion beam tracking using ultrasound motion detection
}

\author{
M. Prall, ${ }^{\text {a) }}$ R. Kaderka, N. Saito, C. Graeff, C. Bert, ${ }^{\text {b) }}$ and M. Durante \\ GSI Helmholtzzentrum für Schwerionenforschung GmbH, Planckstraße 1, 64291 Darmstadt, Germany \\ K. Parodic) \\ Heidelberg University Hospital, Department of Radiation Oncology, Im Neuenheimer Feld 400, 69120 \\ Heidelberg, Germany
}

J. Schwaab, C. Sarti, and J. Jenne

Mediri GmbH, Vangerowstraße 18, 69115 Heidelberg, Germany

(Received 4 September 2013; revised 26 February 2014; accepted for publication 28 February 2014; published 20 March 2014)

Purpose: The use of motion mitigation techniques such as tracking and gating in particle therapy requires real-time knowledge of tumor position with millimeter precision. The aim of this phantombased study was to evaluate the option of diagnostic ultrasound (US) imaging (sonography) as real-time motion detection method for scanned heavy ion beam irradiation of moving targets.

Methods: For this pilot experiment, a tumor surrogate was moved inside a water bath along twodimensional trajectories. A rubber ball was used for this purpose. This ball was moved by a robotic arm in two dimensions lateral to the heavy ion beam. Trajectories having a period of $3 \mathrm{~s}$ and peak to peak amplitude of $20 \mathrm{~mm}$ were used. Square radiation fields of $3 \times 3 \mathrm{~cm}^{2}$ were irradiated on radiosensitive films with a $200 \mathrm{MeV} / \mathrm{u}$ beam of calcium ions having a FWHM of $6 \mathrm{~mm}$. Pencil beam scanning and beam tracking were employed. The films were attached on the robotic arm and thus moved with the rubber ball. The position of the rubber ball was continuously measured by a US tracking system (Mediri GmbH, Heidelberg) and sent to the GSI therapy control system (TCS). This position was used as tracking vector. Position reconstruction from the US tracking system and data communication introduced a delay leading to a position error of several millimeters. An artificial neural network (ANN) was implemented in the TCS to predict motion from US measurements and thus to compensate for the delay.

Results: Using ANN delay compensation and large motion amplitudes, the authors could produce irradiation patterns with a few percent inhomogeneity and about $1 \mathrm{~mm}$ geometrical conformity.

Conclusions: This pilot experiment suggests that diagnostic US should be further investigated as dose-free, high frame-rate, and model-independent motion detection method for scanning heavy ion beam irradiation of moving targets. (C) 2014 American Association of Physicists in Medicine. [http://dx.doi.org/10.1118/1.4868459]

Key words: motion detection, diagnostic ultrasound, hadron therapy, moving tumors

\section{INTRODUCTION}

In state of the art particle therapy of tumors, beam scanning is often preferred due to improved target conformity. Scanned particle beams ${ }^{1,2}$ and target motion interfere if target motion is not accounted for. This interplay leads to deterioration of the dose distribution. ${ }^{3}$ However, beam scanning allows compensation of intrafractional motion via motion mitigation techniques, such as gating or tracking. ${ }^{3,4}$ Real-time knowledge of the target position with millimeter precision is a requirement for these techniques. The aim of our study was to investigate the possibility of using real-time US motion detection for tracking large-amplitude target motion using a scanned heavy ion beam and a simple target geometry.

\section{MATERIAL AND METHODS}

\section{A. Experimental setup}

In our experiment, a radiosensitive film served as twodimensional target. To simulate target motion, the film was continuously moved by a robotic arm (KUKA KR5 sixx R850 by KUKA Roboter, Germany) in a plane perpendicular to the beam. During irradiation, the motion was compensated via the beam tracking technique. ${ }^{1,4}$ The tracking vector was determined using US measurements. Figure 1 shows a schematic of our experimental setup. The $E=200 \mathrm{MeV} / \mathrm{u}$ and $\mathrm{FWHM}=6 \mathrm{~mm}$ calcium beam was perpendicular to both the motion and film plane. A rubber ball $(d=2 \mathrm{~cm}$ diameter) and exchangeable sheets of radiosensitive film (Kodak X-Omat) were attached to a common plexiglas fixture keeping their relative position constant. This fixture was moved by the robotic arm ${ }^{5}$ along two-dimensional motion trajectories perpendicular to the beam (up-down and rightleft in beam's eye view with respect to the room coordinate system). The right-left position of the fixture was continuously measured by a laser distance sensor (SICK, type OD 100-35P840) having a precision better than $0.1 \mathrm{~mm}$ and a negligible delay (below $1 \mathrm{~ms}$ ). The measurement values of the laser sensor were used as reference for tests of timing and precision (cf. Sec. 3.A). For continuous motion correction of ion beam delivery, the position of the rubber 


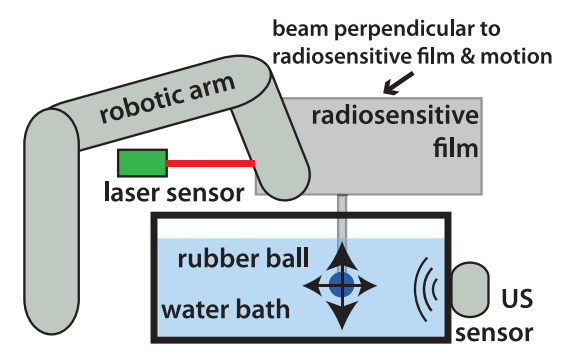

FIG. 1. Experimental setup. The beam was perpendicular to the radiosensitive film and the plane in which the film was moved. The US sensor was used to determine the $2 \mathrm{D}$ position of the rubber ball in a parallel plane. The rubber ball and the film were mechanically coupled and thus moved together. The laser sensor measured the right-left position.

ball was measured using an US tracking system described below.

\section{B. Ultrasound motion detection}

Positions are determined from US measurements in realtime by the Sonoplan II US tracking system (Mediri GmbH, Heidelberg, Germany). The US-tracking system based on a DiPhAS (digital phase Array System) US imager system (Fraunhofer IBMT, St. Ingbert, Germany) comprises the US beam former, a conventional diagnostic US probe and a specialized US tracking probe. The tracking probe includes two $5.5 \mathrm{MHz}, 64$ element phased array ultrasound transducers spanning up two perpendicularly oriented US image planes which define the limits of the tracking volume, such that quasi-3D tracking is possible. The tracking software analyzes the US stream (3-4 ms/slice) and calculates the position of manually pre-defined contours, i.e., structures such as the diaphragm, organ borders, vessels, or in our case the rubber ball phantom (cf. Fig. 2). The tracking algorithm uses active contours and conditional density propagation ${ }^{6}$ as well as the different intensity values of the US images to calculate the actual target contour. Beside the 2D-translation, the tracking algorithm analyzes the rotation as well as the $2 \mathrm{D}$ scaling of the contour. $^{7}$

In the presented experiment, the US software was running on a dedicated computer determining the positions of the rubber ball in both US planes. The 2D position of the 2D plane perpendicular to the beam was sent via network (UDP protocol) directly to the GSI therapy control system (TCS). ${ }^{8}$ In

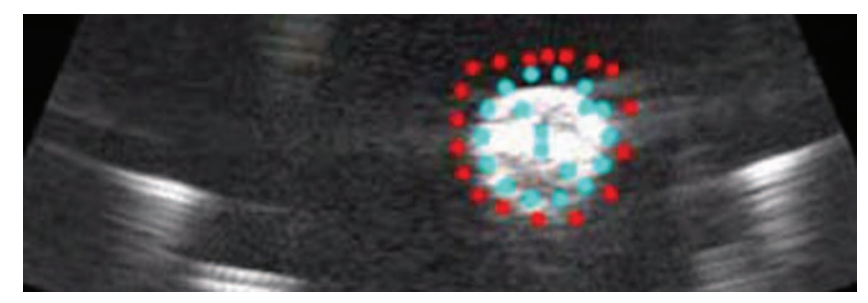

FIG. 2. Screenshot of a US image. The two concentric rings of dots were manually created to specify the approximate borders of the rubber ball as input for the tracking algorithm. this experiment, the relative coordinate information that was produced by the US tracking system was processed by the TCS. An arbitrary origin close (a few $\mathrm{mm}$ ) to the beam axis was used. It has been demonstrated that the US probe can be attached to a patient and optically tracked in order to yield absolute tracking coordinates. ${ }^{9}$ While the adjustment of the beam position to compensate target motion is a common functionality of the TCS for beam tracking, the use of the US motion monitoring signal as input to this procedure was new.

\section{C. Artificial neural network}

For systematic testing, laser and US data were stored in $\log$ files on the receiver computer of the TCS. An inspection of these $\log$ files has revealed that US position data arrive with a delay of about $\Delta t=200 \mathrm{~ms}$ with respect to the laser (reference) data (cf. Sec. 3.A). During irradiation, we compensated this delay using an artificial neural network (ANN). We used a very basic ANN approach in order to demonstrate the feasibility of the method. Especially irregular motion trajectories require more effort going beyond the scope of this note (see discussion). ANNs are nonlinear computational models which can be used to predict the evolution of a discrete timeseries from previous values..$^{10}$ One of their features is the ability to learn the evolution of such a time-series from example data. In an iterative learning process called training, internal weights are optimized such that the ANN reproduces the timeevolution of the discrete example time-series. For our application, we implemented two independent position predictions (for up-down and left-right) on the receiver computer of the TCS (using ANN code by R. Rao, University of Washington). The internal weights of each ANN were optimized via the backpropagation algorithm in 1000 to 1500 training iterations based on a training time-series containing 200 US measurements. At an US sampling rate of about $s=150 \mathrm{~ms}$, this took about $30 \mathrm{~s}$. Five future positions were computed from the 10 latest US position measurements. In practice, an interpolation between the first $(150 \mathrm{~ms})$ and second ( $300 \mathrm{~ms}$ ) time-discrete output of the ANN was used as prediction. Position predictions were added to the above-mentioned $\log$ files.

\section{D. Measurements and data analysis}

We performed several measurement series with a moving and one with a static target. The static irradiation, for which no US position measurements had to be used for motion compensation, served as reference. In the other series, the target was moved simultaneously in both (left-right and up-down) directions. We used three different trajectories $T_{i}(t)$ $=A \cdot \sin ^{n}\left(\frac{2 \pi}{T} t+\varphi_{i}\right)$ with $n=1,2,4$ and $\varphi_{i}=0$. $A$ was chosen such that the peak to peak amplitude always was $20 \mathrm{~mm}$. The index $i$ symbolizes the two motion coordinates. The period was always $3 \mathrm{~s}$, implying $T=3 \mathrm{~s}$ for $n=1$ and $T=6 \mathrm{~s}$ for $n=2,4$. These curves are known to model diaphragm motion due to breathing. ${ }^{11}$ For each trajectory, measurements 
were made with and without ANN prediction. In each of the measurement series homogeneous $3 \times 3 \mathrm{~cm}^{2}$ square radiation fields were used to irradiate the films with a scanned beam. Irradiated films were developed and digitized with $59 \mathrm{px} / \mathrm{cm}$ $(1 \mathrm{px} \hat{=} 0.17 \mathrm{~mm})$ resolution and 16 bit depth using a medical film scanner (Vidar DosimetryPro Advantage). For quantitative comparisons we determined the average FWHM and the inhomogeneity $(\mathrm{IH})$ of the irradiation patterns. The average FWHM of the blackening was determined from four equidistant lines (spacing $=4.25 \mathrm{~mm}$ ) across each pattern in both dimensions. The inhomogeneity $\mathrm{IH}=\sigma / G$ of the blackening is given by the standard deviation $\sigma$ of the grey values divided by the average of the grey value $G$ in a $17 \mathrm{~mm} \times 17 \mathrm{~mm}$ evaluation box. The dimensions of this evaluation box were determined using the static case (cf. Fig. 4) alone. In this case, there is a central part in which the grey values are almost constant, a plateau. The evaluation box completely fits into this plateau.

\section{RESULTS}

\section{A. Tracking data analysis}

The logged US and laser (reference) data were used to study the precision and timing of our method offline. The US measurement values vary with a root mean square (RMS) of $0.3 \mathrm{~mm}$ around a sinusoidal fit. This indicates the relative uncertainty of US position data. Sinusoidal fits to laser (true position) and US data exhibit a relative phase shift of $\Delta \phi=24^{\circ}$ which corresponds to a delay of $\Delta t=200 \mathrm{~ms}$. The position error resulting from this delay was compensated using the ANN. Note that this delay corresponds to US motion detection in 3D. The position was determined in 3D (i.e., in both US planes), but only coordinates of a single plane were sent to the TCS for our pilot experiment. The deviation from the fit to the laser (reference) signal with and without ANN prediction is shown in two histograms (Fig. 3). Note that the overall errors, resulting from both the ANN prediction and

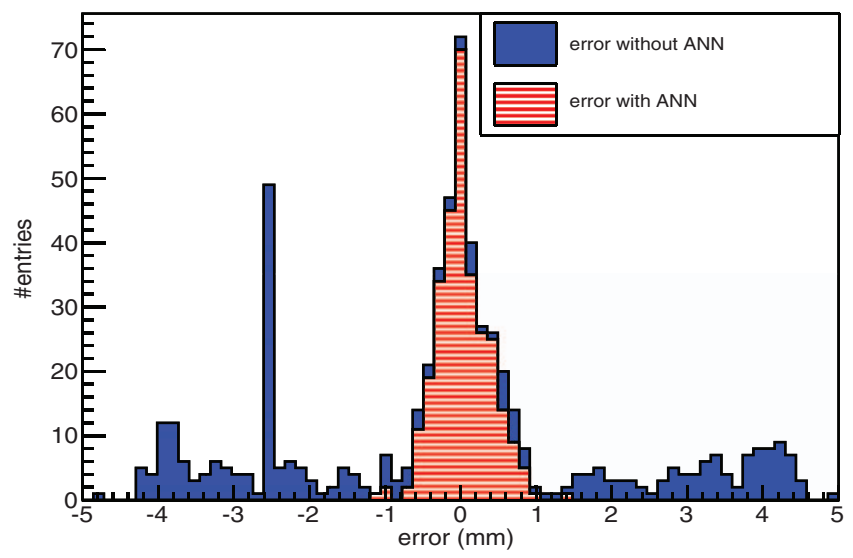

FIG. 3. Performance of the ANN prediction. These two histograms illustrate the deviation of the US data (blue, filled) and ANN predictions (red, hatched) from a sinusoidal fit to the laser data. This fit corresponds to the true position. Note that the overall errors, resulting from both the ANN prediction and US measurement are displayed.

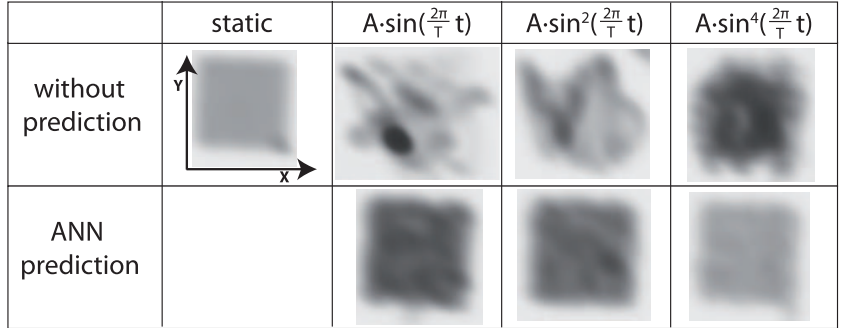

FIG. 4. Irradiated films. Top: Without ANN. Bottom: With ANN. All patterns are shown with the same scale. Note the coordinates used for our numerical analysis (cf. Table I).

US measurement are displayed. Without ANN compensation, US data (blue, filled) scatter with RMS $=2.9 \mathrm{~mm}$ around the laser (reference) data. This scatter is reduced to RMS $=0.3 \mathrm{~mm}$ by the ANN (red, hatched). This is comparable to the relative uncertainty of US itself. For the case $\sin ^{4}$ comparable results were found: US data scattered around a fit curve with RMS $=0.5 \mathrm{~mm}$. Scatter around the laser (reference) was reduced from $2.8 \mathrm{~mm}$ to $0.6 \mathrm{~mm}$ by ANN position prediction. The axis not monitored by the laser can be assumed to have the same delay. The US algorithm determines both pairs of coordinates in one calculation. The coordinates used for motion compensation are sent to the TCS in a common data package.

\section{B. Beam delivery analysis}

Figure 4 shows the irradiation patterns produced in the experiments with radiographic films. The figure shows that ANN prediction notably improves homogeneity and shape of the patterns.

It is clearly visible that the ANN and static irradiation patterns have different absolute blackening. Experimental conditions did not allow to set the absolute beam intensity for the individual irradiations. Therefore our numerical analysis was based on relative, not absolute numbers. Based on film blackening, we have computed numerical results (cf. Table I) to give the reader a quantitative counterpart to the visual impression of Fig. 4. As all patterns are produced

TABLE I. Numerical results: Homogeneity and conformity.

\begin{tabular}{|c|c|c|c|c|}
\hline \multicolumn{5}{|c|}{ Static target } \\
\hline & FWHM X(mm) & & $32.2 \pm 0.1$ & \\
\hline & FWHM Y(mm) & & $32.5 \pm 0.1$ & \\
\hline & $\mathrm{IH}(\%)$ & & 0.8 & \\
\hline & Moving targets & $A \cdot \sin$ & $A \cdot \sin ^{2}$ & $A \cdot \sin ^{4}$ \\
\hline$\overline{\text { No }}$ & FWHM X (mm) & $24 \pm 5$ & $19 \pm 10$ & $36 \pm 2$ \\
\hline ANN & $\begin{array}{c}\text { FWHM Y }(\mathrm{mm}) \\
\text { IH (\%) }\end{array}$ & $\begin{array}{c}24 \pm 4 \\
>15\end{array}$ & $\begin{array}{c}26 \pm 6 \\
>18\end{array}$ & $\begin{array}{c}37 \pm 2 \\
>13\end{array}$ \\
\hline With & FWHM X (mm) & $33.5 \pm 0.5$ & $33.1 \pm 0.5$ & $31.3 \pm 0.5$ \\
\hline ANN & FWHM Y (mm) & $34.1 \pm 0.5$ & $33.4 \pm 0.5$ & $31.9 \pm 0.5$ \\
\hline & $\mathrm{IH}(\%)$ & 5.5 & 6.3 & 3.5 \\
\hline
\end{tabular}


with Gaussian beam spots (FWHM $=6 \mathrm{~mm})$, the static (reference) pattern does not have an infinitely sharp edge resulting in a width of FWHM $\approx 32 \mathrm{~mm}$. The FWHM of the patterns produced without ANN prediction have ragged edges which manifests itself in a large uncertainty (up to $10 \mathrm{~mm}$ ) of the FWHM and a large deviation (up to about $13 \mathrm{~mm}$ ) of the FWHM from the static value. As already mentioned there is no clear center/plateau for the moving cases without ANN prediction. In these cases, we varied the position of the evaluation box to estimate a rough lower limit for IH. On the other hand, if ANN prediction is employed, the edges of the squares become straight (uncertainty of FWHM $=0.5 \mathrm{~mm}$ ) and the width of the patterns is comparable to the static case (difference about $1 \mathrm{~mm}$ ). The inhomogeneity is reduced to a few percent but still larger than in the static case $(0.8 \%)$.

\section{DISCUSSION}

In our experiment, we quickly moved a tumor-surrogate in a water tank with large amplitudes and constantly measured its position using US. This position was used as tracking vector for the irradiation of radiosensitive films. In our experimental setup, the US-tracking system could measure the position of the rubber ball with a few tenths of a millimeter precision and a sampling rate of about $f=6.5 \mathrm{~Hz}$. Our log file analysis revealed a delay of about $\Delta t=200 \mathrm{~ms}$. One contribution to this delay is the finite speed of US in water $(v=1540 \mathrm{~m} / \mathrm{s})$ resulting in about $\Delta t_{U S}=8 \mathrm{~ms}$ for 64 lines and about $l=0.1 \mathrm{~m}$ distance between ball and sensor. The computation of the position from the US image takes $\Delta t_{\text {calc }}=2-4 \mathrm{~ms}$. From previous measurements, we know that the communication delay using UDP network transfer is $\Delta t_{\text {com }}=16 \pm 7 \mathrm{~ms}^{12}$ The contribution from image computation (which was performed for both US planes, i.e., in 3D) was not determined separately. Non-optimized image processing steps of the US-imager (DiPhAS) could be the reason for the residual $\approx 170 \mathrm{~ms}$. Due to our choice of trajectories having a rather large amplitude the overall delay $\Delta t=200 \mathrm{~ms}$ caused a position error of up to a few millimeters. It was compensated using an ANN prediction, whose uncertainty (a few $0.1 \mathrm{~mm}$ ) is comparable with the relative precision of US position measurement. The use of an ANN significantly improved irradiation results. The periodic motion of the tracked phantom was periodic and rather simple. However, one has already shown that US tracking can handle irregular and complex motion. ${ }^{7}$ As our ANN has learned a fixed motion pattern, it would almost certainly have greater difficulty in the case of motion irregularities as in real patients. In its current form, our ANN algorithm will have limited predictive power in the case of motion irregularities. Our ANN learns the features of a specific, regular motion pattern and will therefore predict motion resembling this regular pattern. A computer study ${ }^{13}$ analyzing external/internal correlation has successfully shown that a more sophisticated ANN prediction can cope with typical respiratory irregularities. Irregularities considered were: amplitude and period variations, baseline drifts, phase shifts, hysteresis, and the influence of the heartbeat. The authors conclude that (1) the ANN has to be re-trained regularly and (2) accuracy depends on the ANN architecture. In our case, such a re-training of the ANN could be triggered when a current US measurement and a prediction from past values disagree. In practice, the software could switch between two ANNs running in parallel, one is used for prediction and the other one is re-trained in the background. The ANN architecture best suited for a certain patient could be determined automatically during treatment planning, exploiting data collected by means of 4DCT imaging.

\section{CONCLUSION AND OUTLOOK}

In our experiment, we have shown that US motion detection and beam tracking allow to significantly suppress interplay effects inherent to scanned ion beam RT. We used a simple geometry, both of the tracked object and its trajectory. It has already been demonstrated that the combination of optical tracking of the US sensor position and orientation together with US monitoring can be used for real-time position reconstruction with sub-mm accuracy. ${ }^{9}$ Work is being done to reduce the delay and thus the systematic prediction error. A related study ${ }^{13}$ has demonstrated the robustness of ANN prediction for real-time tumor tracking even if the breathing motion pattern is distorted. Further experiments should investigate our method using more realistic, deformable geometries, motion in 3D and realistic motion patterns, possibly leading towards a dose-free, model-independent and non-invasive motion detection method for ion beam scanning.

\section{ACKNOWLEDGMENTS}

Parts of this work were supported by the EU FP7 Project ENVISION (European NoVel Imaging Systems for ION therapy) under Grant Agreement No. 241851 and by Bundesministerium für Bildung und Forschung (BMBF) as BIO-DISC 5 Project No. 0315726A.

\footnotetext{
a)Electronic mail: m.prall@gsi.de

b) Present address: Universitätsklinikum Erlangen, Universitätsstraße 27, 91054 Erlangen, Germany.

${ }^{c}$ Present address: Fakultät für Physik der LMU München, Lehrstuhl für Experimentalphysik - Medizinische Physik, Am Coulombwall 1, 85748 Garching b. München, Deutschland.

${ }^{1}$ Th. Haberer et al., "Magnetic scanning system for heavy ion therapy," Nucl. Instrum. Methods Phys. Res., Sect. A 330, 296-305 (1993).

${ }^{2}$ E. Pedroni et al., "The $200-\mathrm{MeV}$ proton therapy project at the Paul Scherrer Institute: Conceptual design and practical realization," Med. Phys. 22, 37 53 (1995).

${ }^{3}$ C. Bert et al., "Quantification of interplay effects of scanned particle beams and moving targets," Phys. Med. Biol. 53(9), 2253-2265 (2008).

${ }^{4}$ C. Bert et al., "Motion in radiotherapy: Particle therapy," Phys. Med. Biol. 56, R113-R144 (2011).

${ }^{5} \mathrm{P}$. Steidl et al., "A breathing thorax phantom with independently programmable 6D tumour motion for dosimetric measurements in radiation therapy,” Phys. Med. Biol. 57, 2235-2250 (2012).

${ }^{6} \mathrm{M}$. Isard and A. Blake, "Condensation conditional density propagation for visual tracking," Int. J. Comput. Vision 29, 5-28 (1998).
} 
${ }^{7} \mathrm{X}$. Zhang et al., "Real-time organ tracking in ultrasound imaging using active contours and conditional density propagation," in Medical Imaging and Augmented Reality, Lecture Notes in Computer Science Vol. 6326 (Springer-Verlag Berlin Heidelberg, 2010), pp. 286-294.

${ }^{8} \mathrm{~N}$. Saito et al., "Speed and accuracy of a beam tracking system for treatment of moving targets with scanned ion beams," Phys. Med. Biol. 54, 48494862 (2009)

${ }^{9} \mathrm{~J}$. Schwaab et al., "Calibration of an ultrasound tracking system for moving organs in heavy ion therapy," Abstractband 43. Jahrestagung der DGMP (Deutsche Gesellschaft für Medizinische Physik (DGMP), 2013), pp. 657661 (available URL: http://www.conventus.de/dgmp2012/).
${ }^{10}$ M. J. Murphy et al., "Comparative performance of linear and nonlinear neural networks to predict irregular breathing," Phys. Med. Biol. 51, 59035914 (2006).

${ }^{11}$ E. Lujan et al., "A method for incorporating organ motion due to breathing into 3D dose calculations," Med. Phys. 26, 715-720 (1999).

${ }^{12} \mathrm{G}$. Fattori et al., "TU-A-BRA-08: Integration of optical tracking for organ motion compensation in scanned ion-beam therapy," Med. Phys. 39, 3889 (2012).

${ }^{13} \mathrm{M}$. Seregni et al., "Robustness of external/internal correlation models for real-time tumor tracking to breathing motion variations," Phys. Med. Biol. 57, 7053-7074 (2012) 